\title{
Hybrid Full Frequency Precoding for Integrated Remote Wireless Sensor and Multibeam Satellite Networks
}

\author{
Hongjun $\mathrm{Li}^{1,2}$, Feihong Dong ${ }^{1,2}$, Xiangwu Gong ${ }^{1,3}$, Changliang Deng ${ }^{1}$, Luliang Jia ${ }^{1}$, and Jingchao Wang ${ }^{2}$ \\ ${ }^{1}$ College of Communication Engineering, PLA University of Science and Technology, \\ Nanjing, 210007 -China \\ ${ }^{2}$ Institute of China Electronic System Engineering Company, \\ Beijing, 100141 -China \\ ${ }^{3}$ No.95899 Unit, PLAAF \\ Beijing, 100085 -China \\ [E-mail: lee.hongjun@hotmail.com,dfh_sinlab@hotmail.com, xiangwugong@hotmail.com, \\ luotuociyun@163.com, jiallts@163.com,wangwangjingchao@163.com] \\ *Corresponding author: Hongjun $\mathrm{Li}$
}

Received December 21, 2015; revised March 20, 2016; accepted April 12, 2016; published June 30, 2016

\begin{abstract}
This paper investigates an efficient transmission scheme for the remote wireless sensors to receive information which is rarely discussed in the integrated remote wireless sensor and multibeam satellite networks (IWSMSNs). The networks can be employed to exchange sensing information for emergency scenario, ocean scenario, and so on, which are isolated from available terrestrial networks. As the efficient transmission link is important to the IWSMSNs, we propose a hybrid full frequency (HFF) precoding by taking advantage of frequency reuse and multiple-input multiple-output (MIMO) precoding. Considering energy efficiency and sinks fairness are crucial to transmission link, thus the HFF precoding problems are formulated as transmit power minimization (TPM) and max-min fair (MMF) received signal to interference plus noise ratio (SINR) problems, which can be transformed to indefinite quadratic optimization programs. Then this paper presents a semi-definite programming (SDP) algorithm to solve the problems for the IWSMSNs. The promising potential of HFF for the real IWSMSNs is demonstrated through simulations.
\end{abstract}

Keywords: hybrid full frequency, precoding, multiple-input multiple-output, integrated remote wireless sensor and multibeam satellite networks, semi-definite programming

This work is supported by National Natural Science Foundation of China (61231011, 91338201, 91438109 and 91338106). 


\section{Introduction}

$\mathbf{W}$ ireless sensor networks (WSNs) can be applicated in the potential fields and summarized as: natural disaster warning [1], wildlife monitoring [2], health monitoring [3], target tracking [4], military [5] and other fields. The typical application scenario of remote wireless sensors mainly just transmit back information, while the manned remote wireless sensors require more service as to exchange information in the emergency scenario, ocean scenario, military scenario, etc. For instance, the casualties, medical assistance, meteorological and damage information are exchanged from manned airborne, vehicle and individual sensor nodes (SNs) in the disaster emergency scenario. Manned airborne, seabone, vehicle and individual SNs transmit the electromagnetic spectrum, geographical position and damage information back to the command centers and also especially to acquire the battlefield information unknown in the military. Due to the information transmission isolated from available terrestrial networks and wide coverage, the integrated remote wireless sensor and satellite networks (IWSSNs) are identified as crucial for the scenarios to exchange information at acceptable costs [6].

The characteristics of remote manned WSNs employed in the emergency and military scenarios can be summarized as the following: 1) accumulate sensing information; 2) transmit and also can receive information; 3) terrestrial networks unavailable. MSNs can possess a wide coverage and accurate real-time communication within its coverage which are especially independent of terrestrial infrastructures. Thus the communication link between the remote wireless SNs and remote monitoring hosts (RMHs) can be directly established by the MSNs with potential advantages [7]: 1) geographic unrestricted; 2) quick response; 3) high reliability; 4) strung expandability; 5) global coverage. Satellites were considered crucially important to construct the IWSSNs to satisfy the information transmission for the emergency scenarios [8]. The IWSSNs were also presented for monitoring networks [9]. Obviously, an efficient transmission scheme in the satellite link directly impacts performance of IWSSNs, which also cooperates remote WSNs with satellite networks efficiently.

The rest of the article is organized as follows. Section 2 introduces the related works and contibutions. The integrated remote wireless sensor and multibeam satellite networks (IWSMSNs) architecture and signal model are depicted in section 3, and then the hybrid full frequency (HFF) precoding is presented and formulated as the transmit power minimization (TPM) and max-min fair (MMF) problems. Section 4 designs a semi-definite programming (SDP) algorithm for the HFF precoding, and respectively solves the TPM and MMF problems to profit energy efficiency and sinks fairness. Section 5 shows the summary of simulation results to prove the promising potential of HFF for the real IWSMSNs, and ultimately the conclusions is presented in section 6 .

The notation is adopted as follows: vectors and matrices are represented by bold lowercase and uppercase letters. $C^{m \times n}$ and $R^{m \times n}$ stand for spaces of $m \times n$ complex and real matrices, respectively. The operators $|\cdot|,\|\cdot\|_{F}^{2},(\cdot)^{T}$ and $(\cdot)^{\dagger}$ respectively denote absolute value, 2-norm of Frobenius norm, transpose and conjugate transpose over vectors or matrixes. $\mathrm{E}[\cdot]$ stands for the expected value of a random variable. $\mathbf{A} * \mathbf{B}$ represents Hadamard product of two vectors or matrices. The notation $\mathbf{A} \succ 0$ means that $\mathbf{A}$ is a positive definite matrix. $\operatorname{Tr}(\cdot)$ and $\operatorname{rank}(\cdot)$ denote the trace and rank of a matrix. $\operatorname{diag}\left\{a_{1}, \ldots, a_{N}\right\}$ is an $N$ sized diagonal matrix with $a_{1}, \ldots, a_{N}$ as the diagonal elements. 


\section{Related works and contributions}

\subsection{Integrated Wireless Sensor and Satellite Networks}

WSNs mainly comsist of sensing, data processing and communication components [10], which can monitor, transmit, and process the needed physical data as a low-cost technology for various monitoring scenarios [11]. Furthermore, An efficient and adaptive mutual authentication framework for heterogeneous wireless sensor network-based was studied for practical application [12]. However, SNs transmit information from sinks to monitoring hosts through terrestrial networks which are heavily dependent on terrestrial infrastructure. Yet, it's impossible to transmit information to RMHs for the mergency scenario, ocean scenario where lack available terrestrial infrastructure. Hence, the near-space communication [13][14] and satellite communication networks [15] were presented as the communication components for the WSNs which are independent from terrestrial infrastructure.

The IWSSNs can be seen as one kind of integrated terrestrial and satellite networks. [16] introduced specific performance metrics reference packet loss rate, average packet delay, and energy consumption to evaluate the functionality of hybrid sensor-satellite networks. [17] presented hybrid sensor-satellite networks, sensor-satellite direct communication, connections via a gateway node employing random node layout and cluster-based node layout with data aggregation. [18] formulated a general model to evaluate the IWSSNs capacity to optimize the schedules. The IWSSNs have been studied for many works in architectures, capacity and efficiency analysis, yet there are rarely previous research on transmit scheme for the IWSSNs.

\subsection{Existing Multiple-Input Multiple-Output (MIMO) Technologies}

MIMO technologies have been studied in the WSNs to improve energy efficiency and reduce cost. [19] adopted the cooperative MIMO and data aggregation techniques to reduce the energy consumption in WSNs by decreasing the amount of data for transmission. [20] proposed SNs with MIMO receivers that perform a nonlinear operation on the complex valued received signal, which possessed the low cost, low power and low complexity that were crucial for sensor networks. [21] considered an energy efficient non-overlapping channel MAC to be utilized for WSNs, which improved spectrum efficiency and energy efficiency. [22] exploited the spatial and temporal correlation between sensor data for WSNs, the spatial redundancy of sensor data is reduced by similarity based sub clustering, where closely correlated SNs are represented by a single representative node. It should be noted that MIMO technologies are mainly utilized in the terrestrial WSNs and not involved in the IWSSNs.

Meanwhile, MIMO precoding research has been conducted for the MSNs. [23] investigated the characteristics of the satellite channel which ultimately determined the viability of MIMO over satellite. [24] modeled the forward link channel of MSNs as a MIMO broadcast channel, and proposed a linear precoding with full frequency reuse to improve throughput 20\% compared with conventional 3-color frequency reuse. [25] adopted zero-forcing, regularized zero-forcing and minimum mean square error linear algorithm to design linear precoding and ground based beamforming for MSNs. [26] studied the optimization of linear precoding for MSNs with general linear and nonlinear power constraints, the proposed methods also could be applied to processing in satellite. [27] employed the Shadowed-Rice fading channel model and derived an approximate outage capacity expression for the multibeam mobile satellite system.

However, the MIMO precoding assumed that one terminal was served per beam for each time slot by employing time division multiplexing (TDM), and explicitly stated that a set of 
terminals were served per beam for each time slot as an open problem to be studied [25]-[27]. Precoding for MSNs in the existing research mainly considered throughput maximization as the optimization objective, while TPM problem and MMF problem are the hot research area in the WSNs [20][21] and MIMO systems [28]-[31]. Thus, considering energy consumption is a crucial factor of WSNs, the HFF precoding problem is formulated as TPM and MMF problems. The HFF precoding is proposed to sovle the problem of a set of terminals being served per beam for each time slot and be able to lower the antenna aperture of sinks for the real systems.

\subsection{Contributions}

Therefore, the main contributions of this paper can be summarized as the following.

1) This paper focuses on the manned SNs receive information from RMHs through the MSNs besides transmitting information to RMHs. The efficient transmission scheme is crucial for feasibility of the IWSMSNs.

2) An efficient HFF precoding for the IWSMSNs is proposed for the first time. The HFF precoding mitigates intra-beam interference by employing intra-beam frequency reuse and inter-beam interference by employing inter-beam MIMO precoding. It also makes multiple sinks to be served per beam for each time slot by employing TDM.

3) Considering energy efficiency and sinks fairness for the IWSMSNs, the HFF precoding problem is formulated as TPM and MMF problems which can be transformed to indefinite quadratic optimization programs. This paper presents a SDP algorithm to solve the problems for the IWSMSNs, which is demonstrated through numerical simulations.

\section{Networks architecture and problem formulations}

\subsection{Networks Architecture}

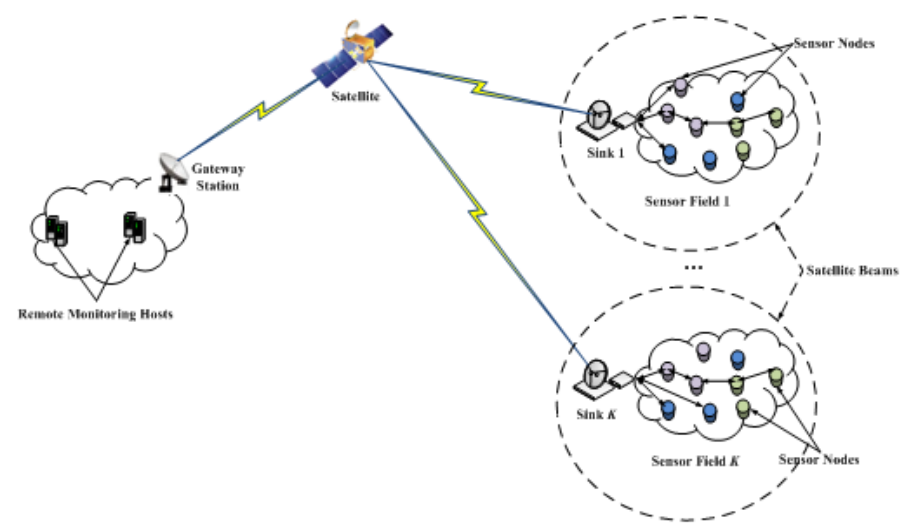

Fig. 1. An integrated remote wireless sensor and multibeam satellite networks architecture.

The architecture of IWSMSNs for the emergency and military scenarios is described in this subsection. As shown in Fig. 1, the IWSMSNs consist of sensor nodes (sensing), remote monitoring hosts (data processing), and multibeam satellite networks (communication components), which are isolated from available terrestrial communication networks. Each beam serves multiple sinks connecting multiple sensor fields. Obviously, MSNs are pivotal to connect SNs and RMHs and an efficient transmission scheme is crucial to the IWSMSNs. The details are depicted in the following. 
1) Sensor nodes: The aim of SNs is to measure dynamic physical quantities for different scenarios, which take inputs from the remote area through corresponding devices and translate the inputs into electrical signals [16]. Signals are digitalized and encapsulated into data packets by analogue-digital converter circuits, and transmitted through MSNs.

2) Remote monitoring hosts: In the majority of WSN cases, RMHs just receive and process packets including audio, video and data from SNs to obtain the comprehensive information. However, the manned SNs also acquire the information unknown from the RMHs in the emergency scenario. That is, RMHs receive/transmit and process data packets from/to SNs through MSNs in the IWSMSNs.

3) Multibeam satellite networks: The MSNs connect SNs with RMHs, i.e., sinks exchange information with SNs and also can be directly utilized as SNs, while RMHs exchange information with gateway station (GS) through satellite link from and to sinks. MSNs consist of a multibeam geosynchronous orbit (GEO) satellite, gateway stations and earth stations called sinks. The GEO satellite possesses the advantages of large coverage, broadband, and high capacity. The multibeam technologies improve the frequency and power efficiency, which increases the system capacity and decreases antenna aperture of sinks to lower the cost.

Therefore, the MSNs are efficient and affordable for the IWSMSNs such as the emergency scenarios. The paper mainly investigates the efficient transmit scheme as: the TPM problem is considered from the perspective of satellite as the satellite is power limited; the MMF problem is considered from the perspective of sinks to guarantee the quality of service (QoS) of sinks with worst SINR.

\subsection{Signal Model}

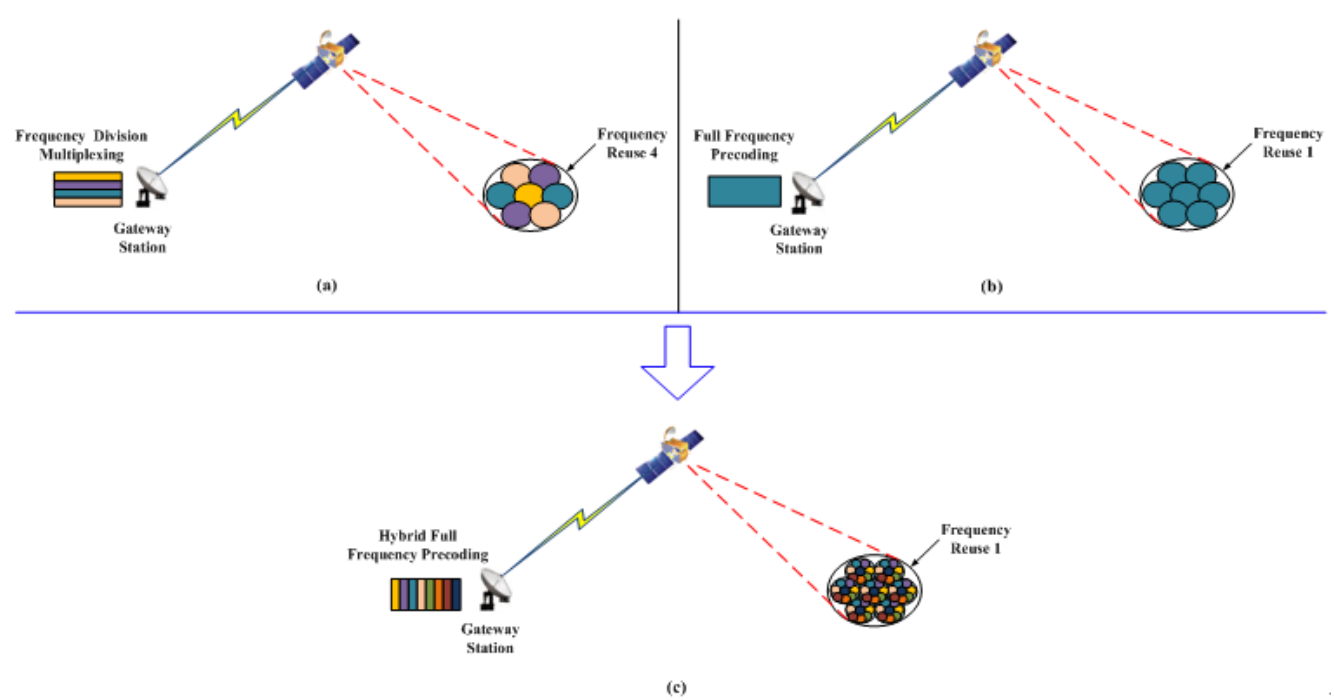

Fig. 2. Transmission schemes for the multibeam satellite networks. (a): Conventional 4-color frequency reuse. (b): Full frequency precoding. (c): Hybrid full frequency precoding.

Fig. 2 depicts transmission schemes for the MSNs. Fig. 2 (a) presents the conventional inter-beam frequency reuse and defines the horizontal bar as frequency division multiplexing. The conventional 4-color frequency reuse divides the total bandwidth equally into 4 and employs frequency division to mitigate inter-beam interference. Fig. 2 (b) shows the full frequency $(\mathrm{FF})$ precoding to mitigate inter-beam interference by employing MIMO precoding and intra-beam interference by TDM [26]. Firstly, the assumption of one terminal was served 
per beam for each time slot leading to problem of multiple terminals being served per beam. Then, user link bandwidth of FF is up to $\mathrm{GHz}$ where terminals were restricted to be equipped larger antenna aperture.

Thus, the paper proposes a HFF precoding by utilizing conventional inter-beam frequency reuse and MIMO precoding as in Fig. 2 (c). The vertical bars are defined as intra-beam frequency reuse to differentiate the inter-beam frequency reuse, the larger reuse factor, the larger number of sinks being served per beam for each time slot. The HFF precoding mitigates intra-beam interference by employing intra-beam frequency reuse and inter-beam interference by employing MIMO precoding and frequency reuse. The intra-beam frequency reuse of HFF makes multiple sinks be served per beam for each time slot, and lower the antenna aperture. Thus, the MIMO precoding of HFF achieves the full frequency reuse. As is shown in Fig. 1, a broadband bent-pipe multibeam satellite with multiple spot beams operated at Ka band is considered for the IWSMSNs. Each beam serves multiple sinks connecting multiple sensor fields. We investigate the efficient transmission scheme from GS to sinks. The assumption of this paper is that the links of RMHs connect GS and SNs connect sinks is ideal, and the GS can get perfect channel state information (CSI) from the backward link.

$M$ is defined as the intra-beam frequency reuse factor, and we assume that $N$ feeds in the satellite transmit $M$ parallel precoding signals from GS to $K$ beams, where $M$ single antenna sinks are served per beam for each time slot. $B_{u}$ denotes the total bandwidth and $B_{u} / M$ stands for the precoding bandwidth. $M$ precoding signals operated at different frequency are also suit to multi-sink MIMO system. In this theory, the $m$ th sink receives the $m$ th precoding signal which means intra-beam frequency reuse and inter-beam full frequency reuse. $M$ sinks can be served per beam for each time slot and $M \times K$ sinks can be served in $K$ beams. Obviously, the assumption of one sink served per beam for each time slot of FF can be transformed by the HFF precoding when intra-beam frequency reuse factor $M=1$. In a word, the HFF precoding improves the sinks capacity and reduces the bandwidth of broadband precoding signals to suit for sinks with small antenna aperture to receive.

The satellite channel mainly under line-of-sight (LOS) subjected to various atmospheric fading effects originating in the troposphere when above $10 \mathrm{GHz}$. Rain attenuation becomes the more dominant factor as the higher frequency and should be considered. The reference model of satellite channel mainly including free space loss, rain attenuation and beam gain pattern. The channel vector for the $m$ th sink in the $k$ th beam is

$$
\mathrm{h}_{k, m}=\frac{\sqrt{\mathrm{b}_{k, m}}}{L_{k, m}} * \mathrm{a}_{k, m}
$$

where $L_{k, m}, \mathrm{~b}_{k, m}$ and $\mathrm{a}_{k, m}$ respectively denote the scalar of free space loss, vectors of beam gain and rain attenuation [32].

The GS transmits symbol data $s_{k, m}$ from RMHs to the $m$ th sink connecting the $m$ th sensor field in the $k$ th beam with $\mathrm{E}\left(\left|s_{k, m}\right|^{2}\right)=1, m=1, \ldots, M$ and $k=1, \ldots, K$ as the index of sinks. Then the $m$ th sink receives and transmits $s_{k, m}$ to the $m$ th sensor field. The $m$ th precoding signal for $K$ beams is

$$
\begin{aligned}
& \mathrm{x}_{m}=\mathbf{w}_{m} \mathrm{~s}_{m} \\
& \mathrm{x}_{m} \in C^{N \times 1}, \mathbf{w}_{m} \in C^{N \times K}, \mathrm{~s}_{m} \in C^{K \times 1}
\end{aligned}
$$

where $\mathrm{s}_{m}=\left\{s_{1, m}, \ldots, s_{K, m}\right\}^{T}$ and $\mathbf{w}_{m}=\left\{\mathrm{w}_{1, m}, \ldots, \mathrm{w}_{K, m}\right\}$. The $m$ th linear precoding matrix $\mathbf{w}_{m}$ weights the symbol data $\mathrm{s}_{m}$ before transmission. $\mathrm{h}_{k, m}$ denotes as the channel vector between 
$N$ feeds and the $m$ th sink in the $k$ th beam, $\mathbf{h}_{m}$ denotes as the channel matrices between $N$ feeds and the $m$ th sink in $K$ beams, then $\mathbf{h}_{m}=\left\{\mathrm{h}_{1, m}, \ldots, \mathrm{h}_{K, m}\right\}$. Sinks receive the $m$ th precoding signal in $K$ beams can be written as

$$
\begin{aligned}
& \mathrm{y}_{m}=\mathbf{h}_{m} \mathbf{w}_{m} \mathrm{~s}_{m}+\mathrm{n}_{m} \\
& \mathrm{y}_{m} \in C^{K \times 1}, \mathbf{h}_{m} \in C^{K \times N}, \mathrm{n}_{m} \in C^{K \times 1}
\end{aligned}
$$

$\mathrm{n}_{m}$ is the noise vector for the $m$ th sink in the $K$ beam, and the vector element $n_{k, m}$ is the independent and identically distributed (i.i.d.) zero-mean Gaussian random noise with power density $N_{0}$. The superposition of all sinks' signals is given by

$$
\begin{aligned}
& \mathbf{x}=\mathbf{W} \mathbf{s} \\
& \mathbf{x}=\left\{\mathbf{x}_{1}^{T}, \ldots, \mathbf{x}_{M}^{T}\right\}^{T}, \mathbf{W}=\operatorname{diag}\left\{\mathbf{w}_{m}\right\}, \mathbf{s}=\left\{\mathrm{s}_{1}^{T}, \ldots, \mathbf{s}_{M}^{T}\right\}^{T} \\
& \mathbf{x} \in C^{N M \times 1}, \mathbf{W} \in C^{N M \times K M}, \mathbf{s} \in C^{K M \times 1}
\end{aligned}
$$

Due to $M$ parallel precoding signals, the precoding matrix $\mathbf{W}$ is a diagonal matrix and $\mathbf{w}_{m}$ is the element. The channel matrix $\mathbf{h}_{m}$ is the element of diagonal matrix $\mathbf{H}$. The total received signals in the $K$ beams can be written as

$$
\begin{aligned}
& \mathbf{y}=\mathbf{H W s}+\mathbf{n} \\
& \mathbf{H}=\operatorname{diag}\left\{\mathbf{h}_{m}\right\}, \mathbf{n}=\left\{\mathbf{n}_{1}^{T}, \ldots, \mathbf{n}_{M}^{T}\right\}^{T} \\
& \mathbf{y} \in C^{K M \times 1}, \mathbf{H} \in C^{K M \times M M}, \mathbf{n} \in C^{K M \times 1}
\end{aligned}
$$

As described above, the $m$ th sink in the $k$ th beam just receives the $m$ th precoding signal, mitigating the same frequency interference inter-beam through precoding while different frequency interference from intra-beam and inter-beam through receiving filter. Therefore, the signal received by the $m$ th sink in the $k$ th beam can be expressed as

$$
\begin{aligned}
\mathrm{y}_{k, m} & =\mathrm{h}_{k, m}^{\dagger} \sum_{j=1}^{K} \mathrm{w}_{j, m} s_{j, m}+n_{k, m} \\
& =\mathrm{h}_{k, m}^{\dagger} \mathrm{w}_{k, m} s_{k, m}+\mathrm{h}_{k, m}^{\dagger} \sum_{j=1, j \neq k}^{K} \mathrm{w}_{j, m} s_{j, m}+n_{k, m}
\end{aligned}
$$

From the signal decomposition, the SINR for the $m$ th sink in the $k$ th beam is

$$
\operatorname{SINR}_{k, m}=\frac{\left|\mathrm{w}_{k, m}^{\dagger} \mathrm{h}_{k, m}\right|^{2}}{\sum_{j=1, j \neq k}^{K}\left|\mathrm{w}_{j, m}^{\dagger} \mathrm{h}_{k, m}\right|^{2}+\frac{B_{u} N_{0}}{M}}
$$

\subsection{Mathematical Formulation of Transmit Power Minimization}

Considering the MSNs as power limited systems, this subsection chooses transmit power minimization as the optimization objective to design the optimal precoding matrix $\mathbf{W}$. Meanwhile, the sinks SINR should be above a reasonable level to guarantee QoS of sinks, and simultaneously keep the co-channel interference in an acceptable requirement. The total transmit power can be expressed as $\|\mathbf{W}\|_{F}^{2}$, then we address $\frac{1}{N}\|\mathbf{W}\|_{F}^{2}$ as the per feed power constraint in the MSNs. The optimal objective can be decomposed from the precoding matrix as

$$
\frac{1}{N}\|\mathbf{W}\|_{F}^{2}=\frac{1}{N} \sum_{m=1}^{M}\left\|\mathbf{w}_{m}\right\|_{F}^{2}=\frac{1}{N} \sum_{m=1}^{M} \sum_{k=1}^{K}\left\|\mathbf{w}_{k, m}\right\|_{F}^{2}
$$


Thus the TPM problem can be mathematically formulated as

$$
\begin{array}{ll}
\min _{\left\{\mathrm{w}_{k, m}\right\}} & \frac{1}{N} \sum_{m=1}^{M} \sum_{k=1}^{K} \|\left.\mathrm{w}_{k, m}\right|_{F} ^{2} \\
\text { s.t. } & \frac{\left|\mathrm{w}_{k, m}^{\dagger} \mathrm{h}_{k, m}\right|^{2}}{\sum_{j=1, j \neq k}^{K}\left|\mathrm{w}_{j, m}^{\dagger} \mathrm{h}_{k, m}\right|^{2}+\frac{B_{u} N_{0}}{M}} \geq t_{k, m} \\
& \sum_{j=1, j \neq k}^{K}\left|\mathrm{w}_{j, m}^{\dagger} \mathrm{h}_{k, m}\right|^{2} \leq \beta \\
\sum_{m=1}^{M} \sum_{k=1}^{K} \mathrm{w}_{k, m}^{\dagger} \mathbf{Q}_{n} \mathrm{w}_{k, m} \leq P_{n}, \quad n=1, \ldots, N & 9(\mathrm{~b})
\end{array}
$$

9(a) represents the TPM optimization objective for per feed averaged. $t_{k, m}, \beta$ and $P_{n}$ respectively denote the acceptable SINR, the allowable interference threshold and the $n$th linear per feed power constraint. The constraint 9(b) shows that the SINR of sinks should be more than the acceptable threshold $t_{k, m}$. The constraint 9(c) indicates that the interference from the co-channel precoding signal ought to be controlled less than the allowable interference threshold as $\beta$. The constraint 9(d) denotes the per feed power constrained by the power amplifier as $P_{n} \cdot \mathbf{Q}_{n}$ is a zero matrix except the $n$th diagonal element being 1 , which expresses the $n$th feed power constraint. The objective function is regard as non-convex and the linear per feed power constraints introduce additional non-convex difficulty. Therefore the non-convex is considered as the main difficulty of this objective function.

\subsection{Mathematical Formulation of Max-Min Fairness}

This subsection considers MMF as the optimal objective for the MSNs from sinks' perspective. We try to maximize the worst sink SINR constrained by per feed power constraints and interference threshold to design precoding vector $\mathrm{w}_{k, m}(\forall k, m)$. The fairness is defined as that the worst sink is maximized as much as possible to guarantee the QoS of every sink under the feed power constrained and interference control, which much liked to solve the problem of "cask effect" to get the defined "fairness" and avoid the polarization of SINR. It can be expressed as

$$
\begin{aligned}
& \max _{\mathrm{w}_{k, m}} \min _{m=1, \ldots, M, k=1, \ldots, K} \frac{\left|\mathrm{w}_{k, m}^{\dagger} \mathrm{h}_{k, m}\right|^{2}}{\sum_{j=1, j \neq k}^{K}\left|\mathrm{w}_{j, m}^{\dagger} \mathrm{h}_{k, m}\right|^{2}+\frac{B_{u} N_{0}}{M}} 10(\mathrm{a}) \\
& \text { s.t. } \sum_{m=1}^{M} \sum_{k=1}^{K} \mathrm{w}_{k, m}^{\dagger} \mathbf{Q}_{n} \mathrm{w}_{k, m} \leq P_{n}, n=1, \ldots, N \quad 10(\mathrm{~b}) \\
& \sum_{j=1, j \neq k}^{K}\left|\mathrm{w}_{j, m}^{\dagger} \mathrm{h}_{k, m}\right|^{2} \leq \beta \quad 10(\mathrm{c}) \\
& \left\|\mathrm{w}_{k, m}\right\|_{F}^{2}>0 \quad 10(\mathrm{~d})
\end{aligned}
$$

10(a) maximizes the worst SINR of sink to achieve the max-min fairness. The constraint 10(b) denotes the feed power constraint $P_{n}$ being same with 9(d). The constraint 10(c) denotes the allowable interference threshold being same with the constraint 9(c). The constraint 10(d) shows that the power of precoding vectors ought to be positive. 


\section{SDP ALGORITHM for HFF PRECODING}

Obviously, the constraints in problem (9) and (10) are non-convex in $\mathrm{w}_{k, m}$. It's difficult to achieve the optimal precoding directly as the problems are NP-hard. This section mainly presents the optimal algorithm design for the TPM problem and MMF problem.

\subsection{SDP Algorithm for Transmit Power Minimization}

The solution of the optimal precoding vector $\mathrm{w}_{k, m}$ can achieve the minimum transmit power in this subsection. The problem in (9) can be seen as a non-convex quadratic problem and resorted to semi-definite relaxation [33]. The relaxation can be obtained by variables change

$$
\left|\mathrm{w}_{k, m}^{\dagger} \mathrm{h}_{j, m}\right|^{2}=\mathrm{w}_{k, m}^{\dagger} \mathrm{h}_{j, m} \mathrm{~h}_{j, m}^{\dagger} \mathrm{w}_{k, m}^{\dagger}=\operatorname{Tr}\left(\mathrm{w}_{k, m}^{\dagger} \mathrm{h}_{j, m} \mathrm{~h}_{j, m}^{\dagger} \mathrm{w}_{k, m}\right)=\operatorname{Tr}\left(\mathrm{w}_{k, m} \mathrm{w}_{k, m}^{\dagger} \mathrm{h}_{j, m} \mathrm{~h}_{j, m}^{\dagger}\right)
$$

The variables substitution is expressed as

$$
\mathbf{W}_{k, m}=\mathrm{w}_{k, m} \mathrm{w}_{k, m}^{\dagger}, \mathbf{H}_{k, m}=\mathrm{h}_{k, m} \mathrm{~h}_{k, m}^{\dagger}
$$

Then the TPM optimal objective (9) can be transformed to

$$
\begin{array}{ll}
\min & \frac{1}{N} \sum_{m=1}^{M} \sum_{k=1}^{K} \operatorname{Tr}\left(\mathbf{W}_{k, m}\right) \\
\text { s.t. } & \operatorname{Tr}\left(\mathbf{W}_{k, m} \mathbf{H}_{k, m}\right)-t_{k, m} \sum_{j \neq k}^{K} \operatorname{Tr}\left(\mathbf{W}_{j, m} \mathbf{H}_{k, m}\right) \geq t_{k, m} \frac{B_{u} N_{0}}{M} \\
& \sum_{j=1, j \neq k}^{K} \operatorname{Tr}\left(\mathbf{W}_{j, m} \mathbf{H}_{k, m}\right) \leq \beta \\
& \sum_{m=1}^{M} \sum_{k=1}^{K} \operatorname{Tr}\left(\mathbf{W}_{k, m} \mathbf{Q}_{n}\right) \leq P_{n}, n=1, \ldots, N . \\
& \mathbf{W}_{k, m} \succ 0 \\
& \operatorname{rank}\left(\mathbf{W}_{k, m}\right)=1, k=1, \ldots, K, m=1, \ldots, M .
\end{array}
$$

The objective function and trace constraints in problem (13) both are linear in $\mathbf{W}_{k, m}$ without considering $\operatorname{rank}\left[\mathbf{W}_{k, m}\right]=1$. Then we firstly expand the feasible solution through abandoning the constraint $\operatorname{rank}\left[\mathbf{W}_{k, m}\right]=1$. The problem in (13) can be relaxed to

$$
\begin{aligned}
& \min \frac{1}{N} \sum_{m=1}^{M} \sum_{k=1}^{K} \operatorname{Tr}\left(\mathbf{W}_{k, m}\right) \\
& \text { s.t. } \operatorname{Tr}\left(\mathbf{W}_{k, m} \mathbf{H}_{k, m}\right)-t_{k, m} \sum_{j \neq k}^{K} \operatorname{Tr}\left(\mathbf{W}_{j, m} \mathbf{H}_{k, m}\right) \geq t_{k, m} \frac{B_{u} N_{0}}{M} \quad 14(\mathrm{a}) \\
& \sum_{j=1, j \neq k}^{K} \operatorname{Tr}\left(\mathbf{W}_{j, m} \mathbf{H}_{k, m}\right) \leq \beta \quad \text { 14(b) } \\
& \sum_{m=1}^{M} \sum_{k=1}^{K} \operatorname{Tr}\left(\mathbf{W}_{k, m} \mathbf{Q}_{n}\right) \leq P_{n}, n=1, \ldots, N . \quad \text { 14(c) } \\
& \mathbf{W}_{k, m} \succ 0, m=1, \ldots, M, k=1, \ldots, K . \quad 14(\mathrm{~d})
\end{aligned}
$$

The problem (14) is regard as a SDP to be solved by the package CVX [34]. Obviously, the optimal solution of problem (14) equal to problem (9) and (13) when $\operatorname{rank}\left[\hat{\mathbf{W}}_{k, m}\right]=1$, and the optimal precoding vector $\hat{\mathbf{W}}_{k, m} \hat{\mathrm{w}}_{k, m}^{\dagger}=\hat{\mathbf{W}}_{k, m}$. The optimal value of problem (14) lower the bound of problem (9) when $\operatorname{rank}\left[\hat{\mathbf{W}}_{k, m}\right]>1$. Then we adopt the method [28][29] to generate the 
optimal solution from the relaxed SDP in problem (14) after the convex optimization problem being solved. When $\operatorname{rank}\left[\hat{\mathbf{W}}_{k, m}\right]=l>1$ and $\hat{\mathbf{W}}_{k, m}$ is obtained, then $\hat{\mathbf{W}}_{k, m}$ can be decomposed to

$$
\hat{\mathbf{W}}_{k, m}=\mathbf{X}_{k, m} \mathbf{Y}_{k, m} \mathbf{X}_{k, m}^{\dagger}
$$

The eigenvectors of $\hat{\mathbf{W}}_{k, m}$ constitute the unitary matrix $\mathbf{X}_{k, m}$, and matrix $\mathbf{Y}_{k, m}$ is a zero matrix except the $i$ th diagonal element being 1 when $i \leq l$. The optimal solution of problem (9) can be solved by

$$
\hat{\mathrm{w}}_{k, m}=\mathbf{X}_{k, m} \mathbf{Y}_{k, m}^{1 / 2} \mathbf{z}_{k, m}
$$

The elements and vector of $\mathrm{z}_{k, m}$ are both assumed as zero-mean unit-variance complex circularly symmetric uncorrelated Gaussian random variables. As the $\hat{\mathrm{w}}_{k, m}$ may be not always feasible, it can be scaled by a coefficient $\sqrt{\eta}$ and defined as [30]

$$
\eta=\max _{m=1, \ldots, M, k=1, \ldots, K} \frac{t_{k, m}\left(\sum_{j=1, j \neq k}^{K}\left|\mathrm{w}_{j, m}^{\dagger} \mathrm{h}_{k, m}\right|^{2}+\frac{B_{u} N_{0}}{M}\right)}{\left|\mathrm{w}_{k, m}^{?} \mathrm{~h}_{k, m}\right|^{2}}
$$

The novel candidate $\hat{\mathrm{w}}_{k, m}=\sqrt{\eta} \hat{\mathrm{w}}_{k, m}$ is obtained to satisfy all the QoS constraints. Meanwhile, the novel candidate should also check the allowable interference constraint. $\hat{\mathrm{w}}_{k, m}$ ought to be induced into problem (9) to compute the transmit power and choose the smallest value as the optimal solution. Table 1 shows the design of the SDP algorithm for the proposed transmit power minimization problem. The objective function $f\left(\mathbf{W}_{k, m}\right)=\frac{1}{N} \sum_{m=1}^{M} \sum_{k=1}^{K} \operatorname{Tr}\left(\mathbf{W}_{k, m}\right)$ is defined for simplified. The input of the algorithm is the channel matrix $\mathbf{H}_{k, m}$, while the outputs of the algorithm are the precoding vector $\hat{\mathbf{W}}_{k, m}$ and transmit power $f\left(\mathbf{W}_{k, m}\right)$. This paper chooses the initial precoding vector $\hat{\mathrm{w}}_{k, m}(0)$ is taken from the normalized $k$ th column of $\max _{\mathbf{h}_{m}^{\dagger} \mathbf{h}_{m}} E\left[\mathbf{h}_{m}^{\dagger} \mathbf{h}_{m}\right]$. We can analyze that the transmit power will decrease progressively with the iteration of precoding vector $\hat{\mathrm{w}}_{k, m}(i)$, and ultimately obtain the convergence numerical value.

Table 1. Algorithm 1: SDP Algorithm for Transmit Power Minimization Problem

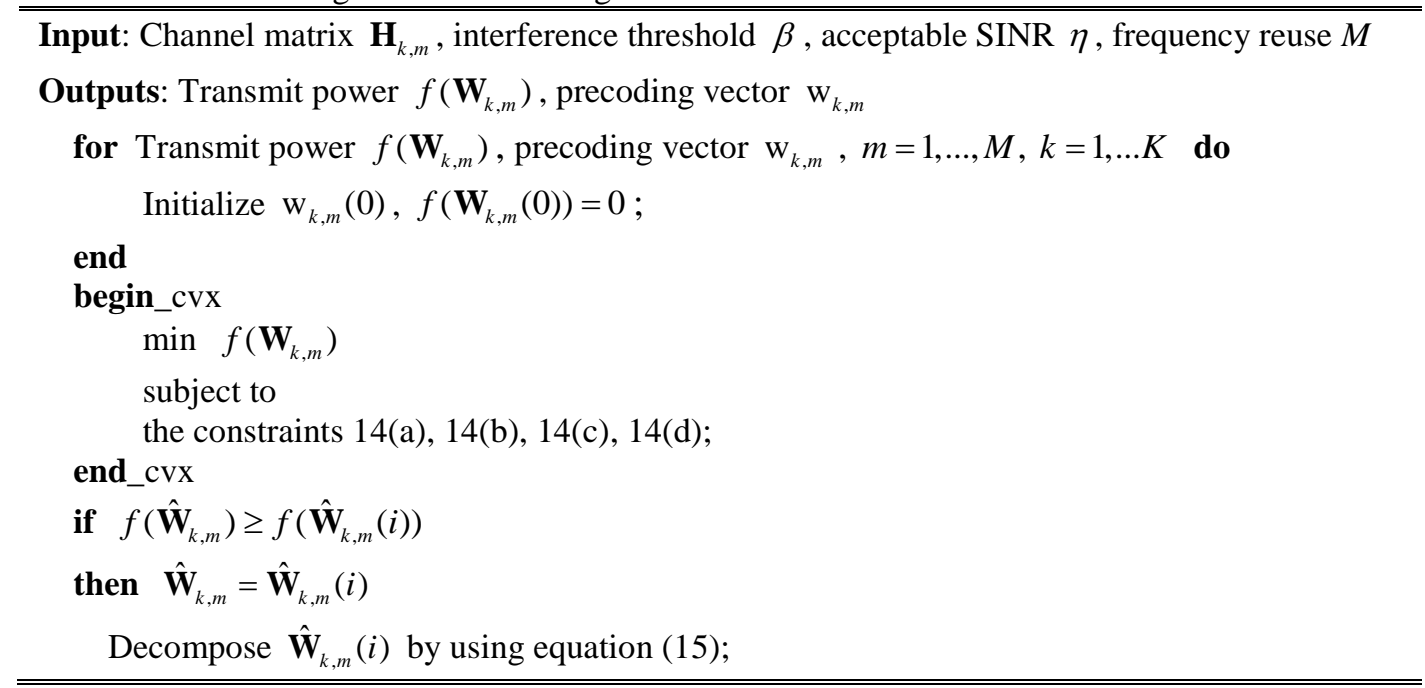


Generate the candidates $\hat{\mathrm{w}}_{k, m}(i)$ by using equation (16);

Find the most violated constraint for $\hat{\mathrm{w}}_{k, m}(i)$;

Scale $\hat{\mathrm{w}}_{k, m}(i)$ by coefficient $\sqrt{\eta}$ of equation (17) to make constraints satisfy with equality;

Select $\hat{\mathrm{w}}_{k, m}(i)$ with the optimal objective;

else Compare $f\left(\hat{\mathrm{W}}_{k, m}\right)$ with $f\left(\hat{\mathrm{W}}_{k, m}(i+1)\right)$ continue

end

for $m, k, m=1, \ldots, M, k=1, \ldots K$ do

$\hat{\mathbf{w}}_{m}(i)=\left\{\hat{\mathbf{w}}_{1, m}(i), \ldots, \hat{\mathbf{W}}_{K, m}(i)\right\}, \hat{\mathbf{W}}(i)=\operatorname{diag}\left\{\hat{\mathbf{w}}_{m}(i)\right\} ;$

end

\subsection{SDP Algorithm for Max-Min Fairness}

To tackle difficulty of non-convex objective function and nonlinear power constraints, by introducing a new variable $\gamma$, the epigraph formulation of problem (10) can be rewritten as

$$
\begin{array}{ll}
\max _{\mathrm{w}_{k, m}} & \gamma \\
\text { s.t. } & \frac{\left|\mathrm{w}_{k, m}^{\dagger} \mathrm{h}_{k, m}\right|^{2}}{\sum_{j=1, j \neq k}^{K}\left|\mathrm{w}_{j, m}^{\dagger} \mathrm{h}_{k, m}\right|^{2}+\frac{B_{u} N_{0}}{M}} \geq \gamma \\
& \sum_{m=1}^{M} \sum_{k=1}^{K} \mathrm{w}_{k, m}^{\dagger} \mathbf{Q}_{n} \mathrm{w}_{k, m} \leq P_{n}, \forall n \in\{1, \ldots, N\} \\
& \sum_{j=1, j \neq k}^{K}\left|\mathrm{w}_{j, m}^{\dagger} \mathrm{h}_{k, m}\right|^{2} \leq \beta \\
& \left\|\mathrm{w}_{k, m}\right\|_{F}^{2}>0
\end{array}
$$

with variables $\mathrm{w}_{k, m}$ and $\gamma \in R^{+}$. It is equivalent to the original problem (10): $\left(\mathrm{w}_{k, m}, \gamma\right)$ is optimal for (18) if and only if $\mathrm{w}_{k, m}$ is optimal for (10) with $\gamma=\gamma_{\max }$. The objective function of the epigraph form problem is a linear function of the variables $\mathrm{w}_{k, m}$ and $\gamma$. The epigraph form problem (18) can be interpreted geometrically as an optimization problem in the 'graph space' $\left(\mathrm{w}_{k, m}, \gamma\right): \gamma$ is maximized over epigraph of (10) and subjected to constraints on $\mathrm{w}_{k, m}$.

Problem (18) belongs to the non-convex quadratically constrained quadratic problem (QCQP), and semi-definite relaxation (SDR) has been proved to reduce non-convex QCQP into a relaxed SDP problem by changing the optimization variables (12). With the change variable $\mathbf{W}_{k, m}=\mathrm{w}_{k, m} \mathrm{~W}_{k, m}^{\dagger}$ and $\mathbf{H}_{k, m}=\mathrm{h}_{k, m} \mathrm{~h}_{k, m}^{\dagger}$, the problem (18) can be relaxed to

$$
\begin{aligned}
& \max _{\mathbf{w}_{k, m}} \gamma \\
& \text { s.t. } \operatorname{Tr}\left(\mathbf{W}_{k, m} \mathbf{H}_{k, m}\right)-\gamma \sum_{j=1, j \neq k}^{K} \operatorname{Tr}\left(\mathbf{W}_{j, m} \mathbf{H}_{k, m}\right) \geq \gamma \frac{B_{u} N_{0}}{M} \quad 19(\mathrm{a}) \\
& \sum_{m=1}^{M} \sum_{k=1}^{K} \operatorname{Tr}\left(\mathbf{W}_{k, m} \mathbf{Q}_{n}\right) \leq P_{n}, n=1, \ldots, N . \quad 19(\mathrm{~b}) \\
& \sum_{j=1, j \neq k}^{K} \operatorname{Tr}\left(\mathbf{W}_{j, m} \mathbf{H}_{k, m}\right) \leq \beta \quad \text { 19(c) } \\
& \mathbf{W}_{k, m} \succ 0, m=1, \ldots, M, k=1, \ldots, K . \quad 19(\mathrm{~d})
\end{aligned}
$$


While problem (19) disregards the unit-rank constraints over the new variable and drops the constraint $\operatorname{rank}\left[\hat{\mathbf{W}}_{k, m}\right]=1$, then relaxes to the quasi-convex problem. As shown that optimization problem in (19) is quasi-convex. A series of convex inequalities are adopted as the general approach to express the sublevel sets of a quasi-convex function to solve the quasi-convex optimization. There $\gamma^{*}$ denotes the optimal value $\gamma$ obtained from the quasi-convex optimization problem (19). Then the quasi-convex problem (19) can be transformed to the feasibility problem

$$
\begin{array}{ll}
\text { Find } & \mathbf{W}_{k, m} \\
\text { s.t. } & \operatorname{Tr}\left(\mathbf{W}_{k, m} \mathbf{H}_{k, m}\right)-\gamma \sum_{j=1, j \neq k}^{K} \operatorname{Tr}\left(\mathbf{W}_{j, m} \mathbf{H}_{k, m}\right) \geq \gamma \frac{B_{u} N_{0}}{M} \\
& \sum_{m=1}^{M} \sum_{k=1}^{K} \operatorname{Tr}\left(\mathbf{W}_{k, m} \mathbf{Q}_{n}\right) \leq P_{n}, n=1, \ldots, N . \\
& \sum_{j=1, j \neq k}^{K} \operatorname{Tr}\left(\mathbf{W}_{j, m} \mathbf{H}_{k, m}\right) \leq \beta \\
& \mathbf{W}_{k, m} \succ 0, m=1, \ldots, M, k=1, \ldots, K .
\end{array}
$$

When the problem (20) is feasible, we can get $\gamma^{*} \geq \gamma$. However, as the problem (20) is not feasible, then $\gamma^{*} \leq \gamma$. Thus, the convex feasibility problem (20) can be solved to check whether the optimal value $\gamma^{*}$ of the quasi-convex optimization problem (19) is greater than any given value $\gamma$. We adopt an iterative algorithm by employing bisection method to solve the quasi-convex optimization problem (19) and the convex feasibility problem (20) at each step on the basis of observation [35]. The convex feasibility problem (20) is firstly assumed to be feasible and start with a previously interval $[s, d]$ which is known to contain the optimal value $\gamma^{*}$. Then the convex feasibility problem is solved at the midpoint $\gamma=(s+d) / 2$ to determine $\gamma$ in the lower or upper half of the interval, and update the interval accordingly until the interval is small enough. $s \leq \gamma^{*} \leq d$ ought to be guaranteed at each step which means $\gamma^{*}$ should be contained in the interval $[s, d]$. The length of the interval after $i$ iterations are perform will be $2^{-i}(d-s)$ as the interval be divided in two for each iteration. Therefore, $\left\lceil\log _{2}((d-s) / \sigma)\right\rceil(0<\sigma<<1)$ iterations are exactly required until termination of the algorithm, and also each step ought to solve the convex feasibility problem. Table 2 shows the design of the SDP algorithm for the max-min fair problem. The input of the algorithm is the channel matrix $\mathbf{H}_{k, m}$, while the outputs of the algorithm are the precoding vector $\hat{\mathbf{w}}_{k, m}$ and maximized worst SINR $\gamma$ of sinks. We can analyze that the worst SINR of sinks will increase progressively with the iteration of the precoding vector $\hat{\mathrm{w}}_{k, m}(i)$, and ultimately obtain the convergence numerical value.

Table 2. Algorithm 2: SDP Algorithm for Max-Min Fair Problem

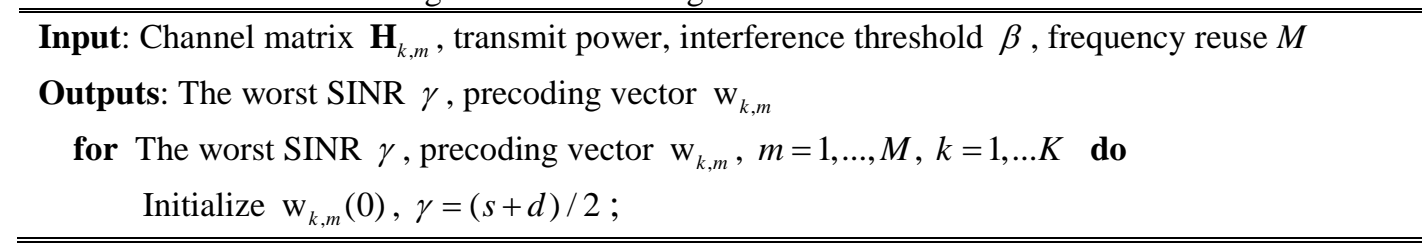




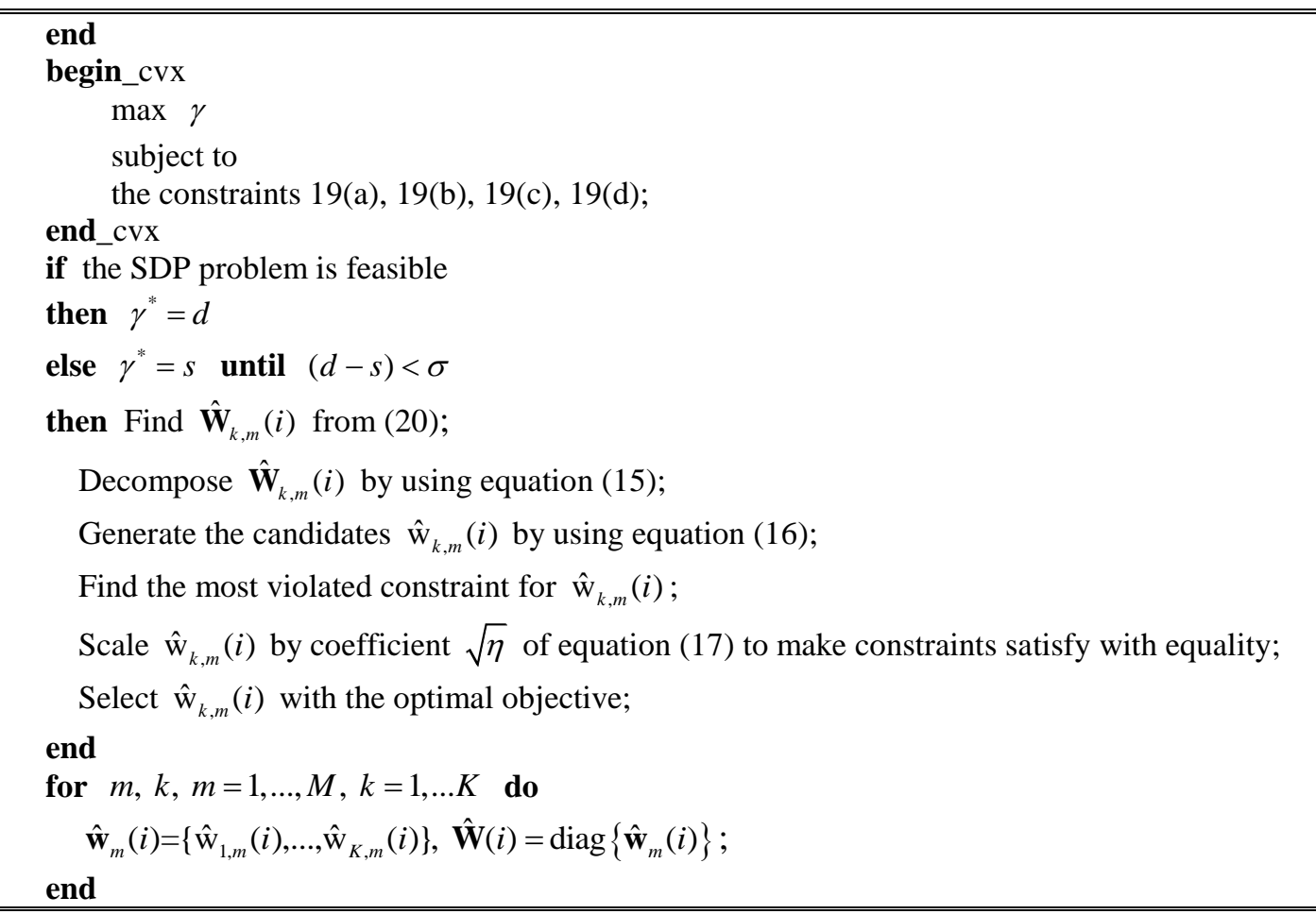

\section{Simulation results}

This section provides the numerical results to verify performance of the HFF precoding. A GEO broadband MSN is considered and Table 3 describes the detail parameters. The feed link is assumed ideal and GS precodes the data symbols from feeds to sinks. Ka band is selected as the sink link frequency, and bandwidth is $500 \mathrm{MHz} . N=9$ feeds are assumed to serve $K=7$ beams and sinks with single antenna, where the number of feeds are considered more than spot beams. The intra-beam frequency reuse factor $M=1,3$ and 6 and $M$ precoding signals are precoded, which means 1, 3 and 6 sinks are served per beam for each time slot. The traveling wave tube amplifier power is assumed to be 80 Watts as the per feed power constraints.

Table 3. Parameters Used in the Simulations and Their Values

\begin{tabular}{|c|c|}
\hline Parameter & Value \\
\hline \hline Orbit & GEO \\
\hline Frequency & $20 \mathrm{GHz}$ \\
\hline Sink link bandwidth & $B_{u}=500 \mathrm{MHz}$ \\
\hline Number of feeds & $K=9$ \\
\hline Number of beams & $M=1,3,6$ \\
\hline Number of sinks per beam for each time slot & $L=7,7 \times 3,7 \times 6$ \\
\hline Allowable interference threshold & $\beta=1,3 \mathrm{~dB}$ \\
\hline Rain attenuation mean & $\mu=-2.6 \mathrm{~dB}$ \\
\hline Rain attenuation variance & $\sigma=1.63 \mathrm{~dB}$ \\
\hline Traveling wave tube amplifier & $80 \mathrm{Watts}$ \\
\hline Conventional inter-beam frequency reuse factor & 4 \\
\hline
\end{tabular}


The performance can be quantified with a benchmark scenario of conventional inter-beam frequency reuse. For the MIMO scenario where the sinks with single antenna, this paper compares performance of HFF respectively with schemes of conventional frequency reuse, zero-forcing (ZF), regularized zero-forcing (R-ZF) and generic iterative algorithm (GIA). ZF, $\mathrm{R}-\mathrm{ZF}$ and GIA precoding are assumed as FF, which means the inter-beam frequency reuse factor $M=1$ of HFF. The numerical analysis used the Matlab embedded by the package CVX. All results are averaged over 1000 simulation runs.

\subsection{Transmit Power Minimization}

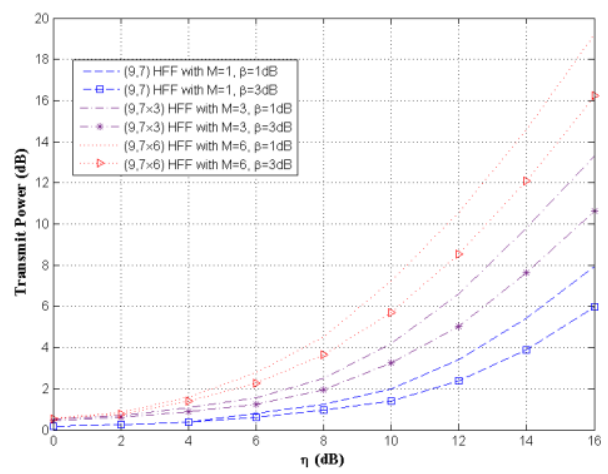

Fig. 3. Algorithm 1: transmit power against SINR threshold $\eta$.

Fig. 3 shows the transmit power per feed averaged against the acceptable SINR $\eta$ in equation (15) of HFF precoding while the allowable interference threshold is selected as $\beta=1 \mathrm{~dB}$ and $\beta=3 \mathrm{~dB}$. The intra-beam frequency reuse factor is respectively selected as $M=1, M=3$ and $M=6$. It's easily to see that HFF will degrade to FF when $M=1$ and one sink is served per beam for each time slot, while 3 and 6 sinks are served per beam for each time slot respectively with $M=3$ and $M=6$. Obviously, the transmit power will increase as the acceptable SINR $\eta$ increases. For the same frequency reuse factor, the transmit power needs more while the $\beta$ decreases from $3 \mathrm{~dB}$ to $1 \mathrm{~dB}$. The transmit power approximate increases about $3.5 \mathrm{~dB}$ from $(9$, 7) to $(9,7 \times 3), 4 \mathrm{~dB}$ from $(9,7 \times 3)$ to $(9,7 \times 6)$ with $\eta=12 \mathrm{~dB}$ and $\beta=3 \mathrm{~dB}$. Fig. 4 shows the transmit power against intra-beam frequency reuse factor $M$ with SINR threshold $\eta=10$ $\mathrm{dB}$. The transmit power needs more as $M$ increases which leads to increase sinks capacity, and differs larger in the higher region. We can conclude that sinks number gain more than transmit power cost to prove the efficiency of algorithm 1. 


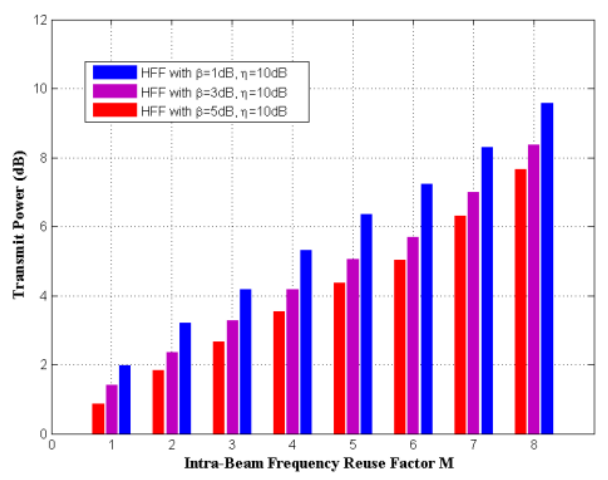

Fig. 4. Algorithm 1: transmit power against frequency reuse factor $M$ with $\eta=10 \mathrm{~dB}$.

Fig. 5 depicts the transmit power per feed averaged against the acceptable SINR $\eta$ of $(9,7)$ HFF compared with conventional inter-beam 4-color frequency reuse, $(9,7) \mathrm{ZF},(9,7) \mathrm{R}-\mathrm{ZF}$ and $(9,7)$ GIA. The allowable interference threshold $\beta=3 \mathrm{~dB}$ and intra-beam frequency reuse $M=1$ are selected for the HFF to satisfy the ZF, R-ZF and GIA of FF. Obviously, the transmit power increases as the acceptable SINR $\eta$ increases. The transmit power against SINR threshold for conventional frequency reuse increases approximate linear. Obviously, the HFF consumes less power under the same $\eta$ while achieves higher $\eta$ under the same transmit power which means the HFF outperforms other four schemes from the perspective of energy efficient. The performance mainly due to the iteration of the precoding vector $\hat{\mathrm{w}}_{k, m}(i)$ which under the constraints. As $\eta=10 \mathrm{~dB}$, the transmit power of HFF approximate less about $0.8 \mathrm{~dB}$ than GIA, $2.5 \mathrm{~dB}$ than ZF and R-ZF, $5.6 \mathrm{~dB}$ than conventional frequency reuse. Sinks number gain of 3 and 6 times for each time slot more than conventional frequency reuse, ZF, R-ZF and GIA of FF Comparing with Fig. 3.

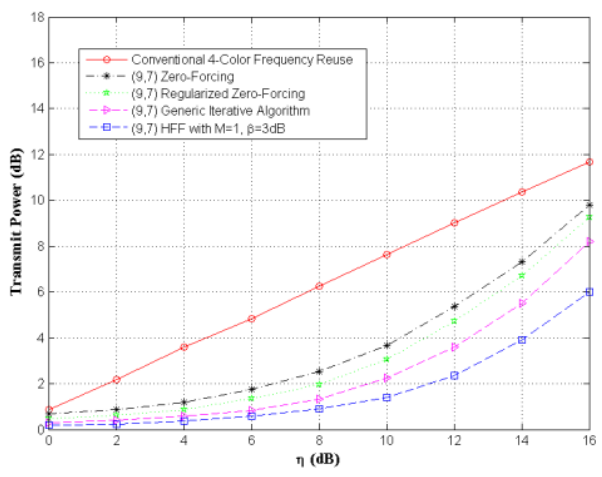

Fig. 5. Transmit power against SINR threshold $\eta$.

Fig. 6 describes the transmit power against the allowable interference threshold $\beta$ with acceptable SINR $\eta=12 \mathrm{~dB}$. It's observed that the transmit power is required less as the allowable interference threshold increase higher with the same intra-beam frequency reuse factor. The transmit power descends clear from interference threshold $\beta=1 \mathrm{~dB}$ to $\beta=3 \mathrm{~dB}$ and unapparent from $\beta=4 \mathrm{~dB}$, which indicates interference threshold above $4 \mathrm{~dB}$ to cause little influence on the transmit power. 


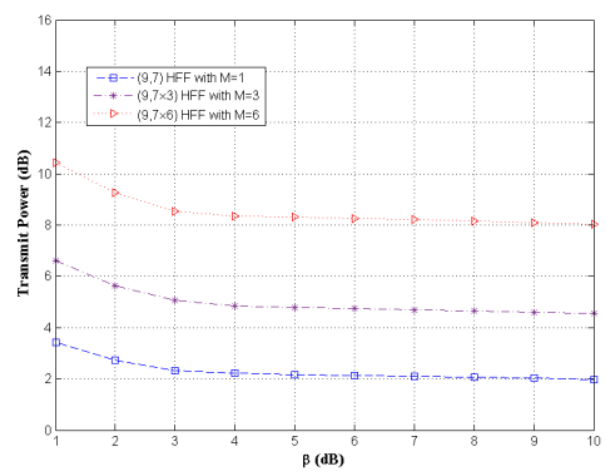

Fig. 6. Algorithm 1: transmit power against interference threshold $\beta$ with $\eta=12 \mathrm{~dB}$.

\subsection{Max-Min Fairness}

Fig. 7 shows the worst sink SINR against the transmit power for the HFF with allowable interference threshold $\beta=1 \mathrm{~dB}$ and $\beta=3 \mathrm{~dB}$. Obviously, performance of the worst sink SINR increases as transmit power increases, especially more obviously when transmit power in the lower level. For the same transmit power and intra-beam frequency reuse factor, the worst sink SINR increases when allowable interference threshold $\beta$ relaxes from $1 \mathrm{~dB}$ to $3 \mathrm{~dB}$. The worst sink SINR drops approximate about $3.8 \mathrm{~dB}$ from $(9,7)$ to $(9,7 \times 3)$ and $3.2 \mathrm{~dB}$ from $(9,7 \times 3)$ to $(9,7 \times 6)$ with transmit power $12 \mathrm{~dB}$ and $\beta=3 \mathrm{~dB}$.

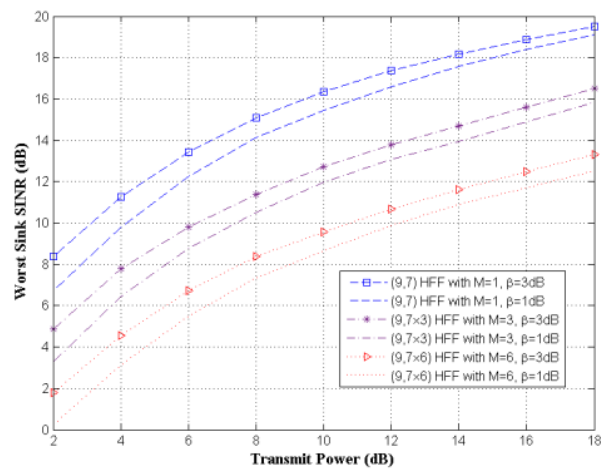

Fig. 7. Algorithm 2: worst sink SINR against transmit power.

Performance of the HFF is influenced by the frequency reuse factor in the MSNs. As the worst sink SINR against intra-beam frequency reuse factor $M$ with transmit power $10 \mathrm{~dB}$ is shown in Fig. 8, the worst sink SINR decreases as $M$ increases, thus the worst sink SINR is in inverse proportion with frequency reuse factor. We can conclude that sinks number gain more than the worst sink SINR loss to prove the efficiency and fairness of algorithm 2. 


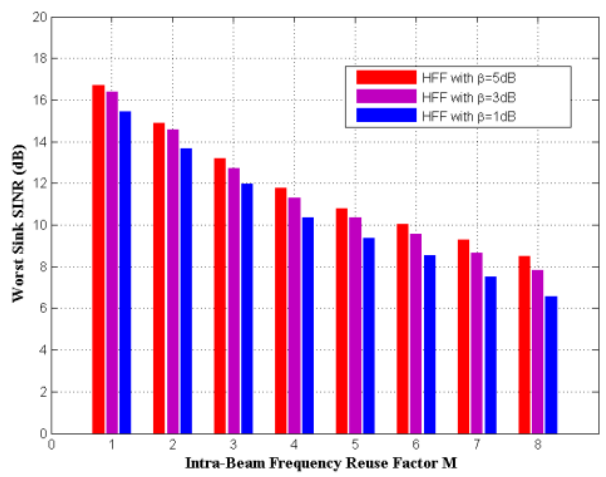

Fig. 8. Algorithm 2: worst sink SINR against frequency reuse factor $M$ with transmit power $10 \mathrm{~dB}$.

Fig. 9 depicts the worst sink SINR against transmit power of the $(9,7)$ HFF compared with conventional inter-beam 4-color frequency reuse, $(9,7) \mathrm{ZF},(9,7) \mathrm{R}-\mathrm{ZF}$ and $(9,7)$ GIA of FF. The HFF achieves higher worst SINR under the same transmit power while consumes less power under the same worst SINR which means the HFF outperforms other four schemes from the perspective of sink fairness and energy efficient. The performance mainly due to the bisection method and iteration of the precoding vector $\hat{\mathrm{w}}_{k, m}(i)$ which under the constraints of problem (20). As the transmit power is $10 \mathrm{~dB}$, the worst sink SINR of HFF outperforms approximate about $0.9 \mathrm{~dB}$ from GIA, $2 \mathrm{~dB}$ from ZF and R-ZF, $5.5 \mathrm{~dB}$ from conventional frequency reuse. The worst sink SINR of conventional frequency reuse increases approximate linear for the principle of power bandwidth balancing.

Fig. 10 shows the worst sink SINR against interference threshold with transmit power 10 dB. For the same transmit power and intra-beam frequency reuse factor, the worst sink SINR increases as the allowable interference threshold increases. The worst sink SINR increases clear from $\beta=1 \mathrm{~dB}$ to $\beta=3 \mathrm{~dB}$ and unapparent from $\beta=4 \mathrm{~dB}$, which indicates interference threshold above $4 \mathrm{~dB}$ to cause little influence on the worst sink SINR. Meanwhile, for the same interference threshold and transmit power, the worst sink SINR becomes lower as the intra-beam frequency reuse factor increases.

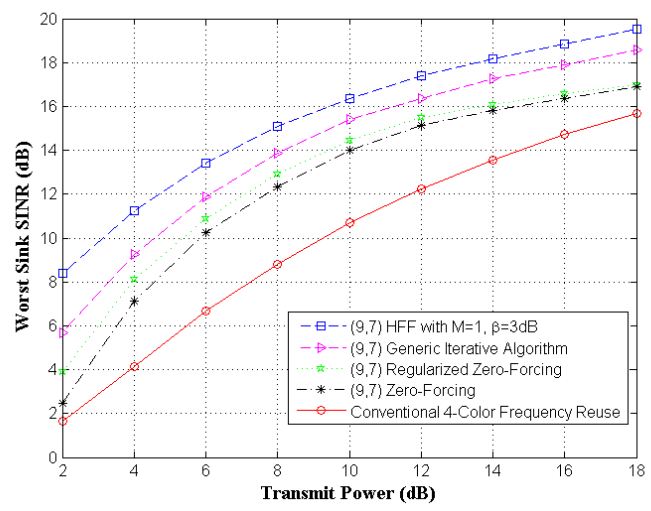

Fig. 9. Worst sink SINR against transmit power. 


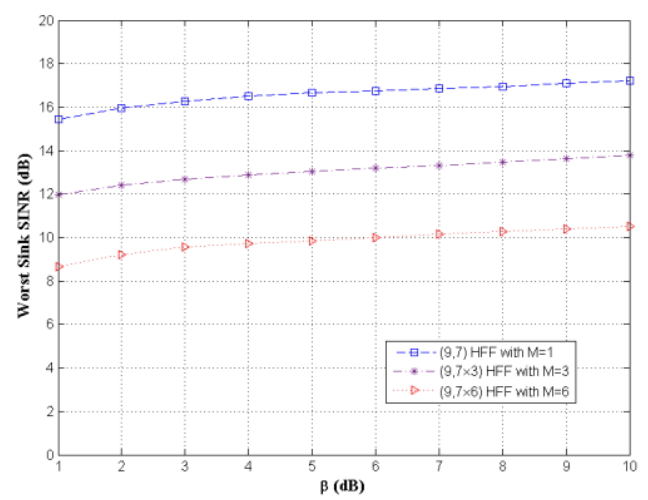

Fig. 10. Algorithm 2: worst sink SINR against interference threshold $\beta$ with transmit power $10 \mathrm{~dB}$.

\section{Conclusions}

This paper has investigated an efficient HFF precoding for the IWSMSNs, which can be employed to exchange sensing information for the emergency scenario, ocean scenario, and so on. By utilizing the frequency reuse and MIMO precoding, the HFF precoding mitigated intra-beam interference by employing frequency reuse and inter-beam by employing MIMO precoding. Considering energy efficiency and fairness are crucial to the IWSMSNs transmission link, the HFF precoding has been formulated as TPM and MMF problems.

Then this paper presented a SDP algorithm to solve the problems of the IWSMSNs. Simulation results showed that energy efficiency and worst SINR of HFF outperform the existing schemes. The HFF increased the capacity of sinks being served per beam for each time slot and decreased the antenna aperture of sinks' to lower cost. The advantages of HFF just coincided with the characteristics of energy limited, large number of sinks, and antenna portable for the IWSMSNs. Meanwhile, the intra-beam frequency reuse factor $M$ affects performance of the HFF, which was in direct proportion with transmit power of TPM and in inverse proportion with the worst sink SINR of MMF. However, the allowable interference threshold $\beta$ was on the contrary and also manifested to cause little influence to the performance above $4 \mathrm{~dB}$. In conclusion, the HFF precoding improved energy efficiency and sinks fairness, and made multiple sinks be served per beam for each time slot. Furhtermore, sinks with small antenna aperture be portable to receive broadband preconding signals which will be a promising solution for real IWSMSNs in the future.

\section{References}

[1] O. Aulov, and M. Halem, "Human sensor networks for improved modeling of natural disasters," IEEE Proceedings, vol. 100, no. 10, pp. 2812-2823, October, 2012. Article (CrossRef Link)

[2] V. Dyo, S. Ellwood, D. Macdonald, A. Markham, et al., "WILDSENSING: design and deployment of a sustainable sensor network for wildlife monitoring," ACM Transactions on Sensor Networks, vol. 8, no. 4, pp. 1651-1654, September, 2012. Article (CrossRef Link)

[3] J. Caldeira, J. Rodrigues, and P. Lorenz, "Intra-mobility support solutions for healthcare wireless sensor networks-handover issues," IEEE Sensors Joural, vol. 14, no. 10, pp. 4339-4348, November, 2013. Article (CrossRef Link) 
[4] G. Zheng, L. Fu, J. Li, and M. Li, "A large-scale multi-track mobile data collection mechanism for wireless sensor networks," KSII Transactions on Internet and Information Systems, vol. 8, no. 3, pp. 857-872, March, 2014. Article (CrossRef Link)

[5] S. Astapov, J. Preden, J. Ehala and V. Milutinovic, "Object detection for military surveillance using distributed multimodal smart sensors," in Proc. of the 19th International Conference on Digital Signal Processing (DSP'14), pp. 366-371, Hong Kong, August 20-23, 2014. Article (CrossRef Link)

[6] I. Akyldiz, W. Su, Y. Sankarasubramaniam and E. Cayirci, "A survey on sensor networks," IEEE Communications Magazine, vol. 40, no. 8, pp. 102-114, August, 2002. Article (CrossRef Link)

[7] J. Mukherjee and B. Ramamurthy, "Communication technologies and architectures for space network and interplanetary internet," IEEE Communications Surveys \& Tutorials, vol. 15, no. 2, pp. 881-897, Second Quarter, 2013. Article (CrossRef Link)

[8] F. Dong, H. Li, X. Gong, Q. Liu and J. Wang, "Energy-efficient transmissions for remote wireless sensor networks: an integrated HAP/satellite architecture for emergency scenarios," Sensors, pp. 22266-22290, vol. 15, no. 9, September, 2015. Article (CrossRef Link)

[9] I. Bisio and M. Marchese, "Satellite earth station (SES) selection method for satellite-based sensor networks," IEEE Communications Letters, vol. 11, no. 12, pp. 970-972, December, 2007. Article (CrossRef Link)

[10] J. Horneber, and A. Hergenroder, "A survey on testbeds and experimentation environments for wireless sensor networks," IEEE Communications Surveys \& Tutorials, vol. 16, no. 4, pp. 1820-1838, Fourth quarter, 2014. Article (CrossRef Link)

[11] L. Borges, F. Velez and A. Lebres, "Survey on the characterization and classification of wireless sensor network applications," IEEE Communications Surveys \& Tutorials, vol. 16, no. 4, pp. 1860-1890, Fourthquarter, 2014. Article (CrossRef Link)

[12] P. Kumar, M. Ylianttila, A. Gurtov, S. Lee and H. Lee, "An efficient and adaptive mutual authentication framework for heterogeneous wireless sensor network-based applications," Sensors, vol. 14, no. 2, pp. 2732-2755, February, 2014. Article (CrossRef Link)

[13] W. Wang, J. Cai, and Q. Peng, "Near-space microwave radar remote sensing: potentials and challenge analysis," Remote Sensing, vol. 2, no. 3, pp. 717-739, March, 2010. Article (CrossRef Link)

[14] F. Dong, M. Li, X. Gong, H. Li, and F. Gao, "Diversity performance analysis on multiple HAP networks," Sensors, vol. 15, no. 7, pp. 15398-15418, June, 2015. Article (CrossRef Link)

[15] W. Wang and D. Jiang, "Integrated wireless sensor systems via near-space and satellite platforms: a review," IEEE Sensors Journal, vol. 14, no. 11, pp. 3903-3914, November, 2014. Article (CrossRef Link)

[16] I. Bisioa and M. Marchese, "Efficient satellite-based sensor networks for information retrieval," IEEE Sensors Journal, vol. 2, no. 4, pp. 464-475, December, 2008. Article (CrossRef Link)

[17] S. Verma, P. Pillai and Y. Hu, "Performance evaluation of alternative network architectures for sensor-satellite integrated networks," in Proc. of the International Conference on Advanced Information Networking and Applications Workshops (WAINA'13), pp. 120-125, Barcelona, Spain, March 25-28, 2013. Article (CrossRef Link)

[18] W. Zhang, G. Zhang, F. Dong, Z. Xie and D. Bian, "Capacity model and constraints analysis for integrated remote wireless sensor and satellite network in emergency scenarios," Sensors, vol. 15, no. 11, pp. 29036-29055, November, 2015. Article (CrossRef Link)

[19] Q. Gao, Y. Zuo, J. Zhang and X. Peng, "Improving energy efficiency in a wireless sensor network by combining cooperative MIMO with data aggregation," IEEE Transactions on Vehicular Technology, vol. 59, no. 8, pp. 3956-3965, October, 2010. Article (CrossRef Link)

[20] G. Psaltopoulos and A. Wittneben, "Nonlinear MIMO: affordable MIMO technology for wireless sensor networks," IEEE Transactions on Wireless Communications, vol. 9, no. 2, pp. 824-832, February, 2010. Article (CrossRef Link)

[21] B. Kim, K. Kim, and B. Roh, "ENC-MAC: energy-efficient non-overlapping channel MAC for cognitive radio enabled sensor networks," KSII Transactions on Internet and Information Systems, vol. 9, no. 11, pp. 4367-4386, November, 2015. Article (CrossRef Link) 
[22] M. Arunraja and V. Malathi, "Collective prediction exploiting spatio temporal correlation (CoPeST) for energy efficient wireless sensor networks," KSII Transactions on Internet and Information Systems, vol. 9, no. 7, pp. 2488-2511, July, 2015. Article (CrossRef Link)

[23] P. Arapoglou, K. Liolis, M. Bertinelli, et al., "MIMO over satellite: a review," IEEE Communications Surveys \& Tutorials, vol. 13, no. 1, pp. 27-51, First Quarter, 2011. Article (CrossRef Link)

[24] L. Cottatellucci, M. Debbah, G. Gallinaro, et al., "Interference mitigation techniques for broadband satellite systems," in Proc. of the 24th AIAA International Communications Satellite Systems Conference (ICSSC'06), pp. 1-13, San Diego, USA, June 11-14, 2006. Article (CrossRef Link)

[25] B. Devillers, A. Perez-Neira and C. Mosquera, "Joint linear precoding and beamforming for the forward link of multi-beam broadband satellite systems," in Proc. of the IEEE Global Telecommunications Conference (GLOBECOM'11), pp. 1-6, Houston, USA, December 5-9, 2011. Article (CrossRef Link)

[26] G. Zheng, S. Chatzinotas and B. Ottersten, "Generic optimization of linear precoding in multibeam satellite systems," IEEE Transactions on Wireless Communications, vol. 11, no. 6, pp. 2308-2320, June, 2012. Article (CrossRef Link)

[27] G. Song, B. Hu and X. Zhang, "Optimal linear precoding scheme for multibeam satellite systems based on partial channel information," in Proc. of the Wireless and Optical Communication Conference (WOCC'15), pp. 1-4, Taipei, Taiwan, October 23-24, 2015. Article (CrossRef Link)

[28] H. Du, T. Ratnarajah, M. Pesavento and C. Papadias, "Joint transceiver beamforming in mimo cognitive radio network via second-order cone programming," IEEE Transactions on Signal Processing, vol. 60, no. 2, pp. 781-792, February, 2012. Article (CrossRef Link)

[29] S. Cai and Y. Gong, "Cognitive beamforming for multiple secondary data streams with individual snr constraints," IEEE Transactions on Signal Processing, vol. 61, no. 17, pp. 4189-4198, September, 2013. Article (CrossRef Link)

[30] T. Phan, A. Vorobyov, N. Sidiropoulos and C. Tellambura, "Spectrum sharing in wireless networks via qos-aware secondary multicast beamforming," IEEE Transactions on Signal Processing, vol. 57, no. 6, pp. 2323-2335, June, 2009. Article (CrossRef Link)

[31] N. D. Sidiropoulos, T. N. Davidson, and Z-Q. Luo, "Transmit beamforming for physical-layer multicasting," IEEE Transactions on Signal Processing, vol. 54, no. 6, June, 2006. Article (CrossRef Link)

[32] D. Christopoulos, S. Chatzinotas, M. Matthaiouy and B. Ottersten, "Capacity analysis of multibeam joint decoding over composite satellite channels," in Proc. of the 45th Asilomar Conference on Signals, Systems and Computers (ASILOMAR'11), pp. 1795-1799, Pacific Grove, CA, November 6-9, 2011. Article (CrossRef Link)

[33] Z. Luo, W. Ma, A. So, Y. Ye and S. Zhang, "Semidefinite relaxation of quadratic optimization problems," IEEE Signal Processing Magazine, vol. 27, no. 3, pp. 20-34, May, 2010. Article (CrossRef Link)

[34] M. Grant, and S. Boyd, Cvx: Matlab software for disciplined convex programming. version 1.21. Article (CrossRef Link)

[35] S. Boyd, and L. Vandenberghe, "Convex optimization," Cambridge University Press, London, United Kingdom, 2009. Article (CrossRef Link) 


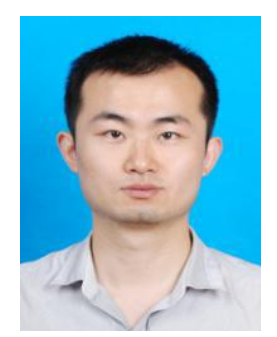

Hongjun Li received his B.S. degree and M.S. degree from College of Communication Engineering, PLA University of Science and Technology, Nanjing, China in 2008 and 2013 respectively. He is currently pursuing his $\mathrm{Ph}$.D degree in College of Communication Engineering, PLA University of Science and Technology. His current research interests include wireless communication, satellite communication and spatial information networks.

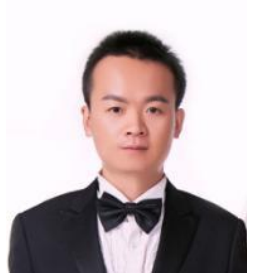

Feihong Dong received the B.E. degree in information system engineering from PLA University of Science and Technology, Nanjing, China in 2010. He is currently working toward the Ph.D. degree with the College of Communications Engineering, PLA University of Science and Technology. His research interests include high altitude platform communication networks, satellite communication networks and space information networks.

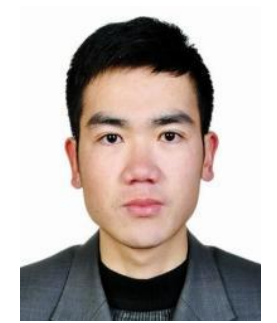

Xiangwu Gong received his B.S. degree from School of Mathematics, Shandong University, China in 2009. He received his M.S. degree from School of Science, PLA University of Science and Technology, Nanjing, China in 2012. He is currently pursuing his Ph.D degree in College of Communication Engineering, PLA University of Science and Technology. His current research interests include wireless communication and physical layer security.

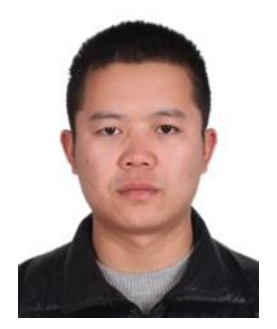

Changliang Deng received his B.S. degree and M.S. degree from College of Communication Engineering, PLA University of Science and Technology, Nanjing, China in 2008 and 2013 respectively. He is currently pursuing his Ph.D degree in College of Communication Engineering, PLA University of Science and Technology. His current research interests include electromagnetic field and microwave technology, wireless communication and blind source separation.

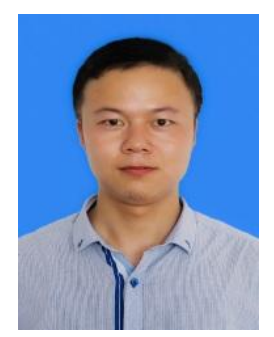

Luliang Jia received his B.S. degree from Lanzhou Jiaotong University, Lanzhou, China in 2010. He received his M.S. degree from College of Communication Engineering, PLA University of Science and Technology, Nanjing, China in 2013. He is currently pursuing his Ph.D degree in College of Communication Engineering, PLA University of Science and Technology. His current research interests include satellite communication and communication anti-jamming technology.

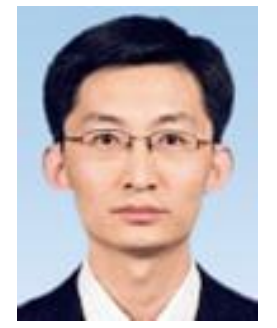

Jingchao Wang received his B.S. degree, M.S. degree and Ph.D degree from Tsing Hua University, Beijing, China in 2004, 2007 and 2011 respectively. He is now with the China Electronic System Engineering Company. His current research interests include satellite communication and space information networks. 